\title{
Optimization of Pulse CVT Based on Improved Particle Swarm Algorithm Zhongkang Song ${ }^{1,{ }^{,}{ }^{*}}$, Peng Wang ${ }^{1, b}$ and Lianqiang $\mathrm{Bai}^{1, \mathrm{c}}$ \\ ${ }^{1}$ Academy of Armored Force Engineering, Beijing 100071, China

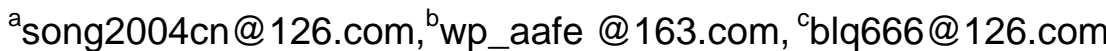

\begin{abstract}
In order to solve the problem that the output speed of the CVT is too high, an optimization of Pulse CVT Based on Improved Particle Swarm Algorithm is proposed. Firstly, an improved particle swarm optimization (PSO) algorithm is proposed, which can improve the accuracy and efficiency of the algorithm by replacing the original velocity and the cognitive part with the chaotic mutation. Secondly, the transmission size parameter is used as the evaluation index of particle swarm optimization algorithm. The fitness function of the pulse continuously variable transmission is established based on the constraints of the length of the rod and the constraint of the transmission angle. Finally, the improved particle swarm optimization algorithm and particle swarm optimization algorithm are used to obtain the optimal solution. The results show that the accuracy and robustness of the improved particle swarm algorithm is improved by an order of magnitude, the solution time is reduced by $70 \%$, and the optimal transmission speed is decreased by about $41 \%$.
\end{abstract}

Keywords: Pulse CVT, Particle Swarm Optimization, Optimization.

\section{Introduction}

Pulse CVT is a kind of transmission mechanism which is composed of pulse generator, speed regulator and overrunning clutch. It is widely used in the fields of agriculture, textile, printing and so on. The fluctuation degree is used to describe the fluctuation degree of the output speed, and it is an important index to measure the performance of the pulsating cvt. Some scholars have carried on the kinematics and kinematics simulation of Zero-Max CVT, and analyzed its motion characteristics, which laid the foundation for the next research [1]. Some scholars further innovate the pulse generator, they designed a new structure of the pulse CVT using planetary gear, cam mechanism and other mechanisms to replace the linkage [2]. The Zero-Max type impulse CVT as the research object, and the degree of fluctuation as the evaluation index, this paper proposes an improved particle swarm algorithm as the optimization method. The mathematical model of the six bar function generator with constraint condition is used as the fitness function, so the size parameter optimization design of the Zero-Max type continuously variable transmission is realized.Do not number your paper: All manuscripts must be in English, also the table and figure texts, otherwise we cannot publish your paper. Please keep a second copy of your manuscript in your office. When receiving the paper, we assume that the corresponding authors grant us the copyright to use the paper for the book or journal in question.

\section{Establishment of mathematical model of pulse generator}

Figure 1 is a type of Zero-Max CVT single-phase pulse generating mechanism of the first and I position of the schematic diagram, the crank $l_{1}$ is an eccentric disc, the connecting rod $l_{3}$ is the speed of the connecting rod, the connecting rod $l_{5}$ is beyond the clutch yoke. The working principle of Zero-Max cvt: The driving shaft drives four eccentric discs $l_{1}$ with phase difference of 90 degrees to rotate at a constant speed, it is converted into the outer yoke ring reciprocating swing by single-phase pulsating parallel mechanism, the clutch filter negative direction swing and merged with approximately uniform rotation to the output shaft of the output pulse. 


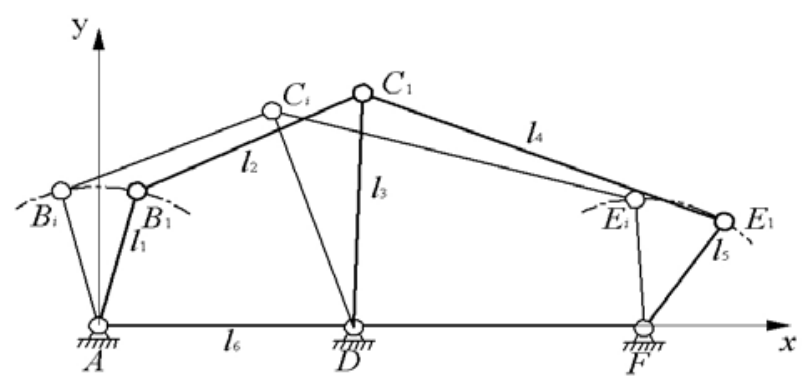

Fig. 1 the Schematic Diagram of Zero-Max CVT

Combined with the current product size, this paper calculates the relationship between the input and output angular function of the single-phase pulse generator, then the input and output angles near the highest speed of the rotor are combined into linear relation. Finally, a set of design variables X are used to determine the minimum square sum of the error of the output curve and the optimal curve through the synthesis of six bar mechanism.

According to the mechanism, B coordinates of the i position can be calculated:

$$
\left(\begin{array}{l}
B_{i} \\
1
\end{array}\right)=\left[D_{A B}\right]\left(\begin{array}{c}
B_{1} \\
1
\end{array}\right)
$$

In the formula, $\left[D_{A B}\right]$ is a general displacement matrix of rigid body $l_{1}$. C,E coordinates can be derived from a similar relationship. According to the conditions that BC, CE rod length is unchanged, the constraint equations:

$$
\begin{aligned}
& {\left[C_{i}-B_{i}\right]^{T}\left[C_{i}-B_{i}\right]=\left[C_{1}-B_{1}\right]^{T}\left[C_{1}-B_{1}\right]} \\
& {\left[C_{i}-E_{i}\right]^{T}\left[C_{i}-E_{i}\right]=\left[C_{1}-E_{1}\right]^{T}\left[C_{1}-E_{1}\right]}
\end{aligned}
$$

The coordinates of $B_{i}, C_{i}, E_{i}$ is brought into the equation group (2); we can get the objective function:

$$
\min F(X)=\sum_{i=2}^{n}\left(\left(U_{3} V_{2}-U_{2} V_{3}\right)^{2}+\left(U_{3} V_{1}-U_{1} V_{3}\right)^{2}-\left(U_{1} V_{2}-U_{2} V_{1}\right)^{2}\right)^{2}
$$

In the formula, $U_{1}=2\left[\left(x_{c 1}-x_{d}\right)\left(x_{d}-x_{b i}\right)+\left(y_{c 1}-y_{d}\right)\left(y_{d}-y_{b i}\right)\right]$;

$$
\begin{aligned}
& U_{2}=2\left[\left(x_{c 1}-x_{d}\right)\left(x_{d}-x_{b i}\right)-\left(y_{c 1}-y_{d}\right)\left(y_{d}-y_{b i}\right)\right] ; \\
& U_{3}=\left(x_{d}-x_{b 1}\right)\left(2 x_{c 1}-x_{d}-x_{b 1}\right)+\left(y_{d}-y_{b 1}\right)\left(2 y_{c 1}-y_{d}-y_{b 1}\right)-\left(x_{d}-x_{b i}\right)^{2}-\left(y_{d}-y_{b i}\right)^{2} ; \\
& V_{1}=2\left[\left(x_{c 1}-x_{d}\right)\left(x_{d}-x_{e i}\right)+\left(y_{e 1}-y_{d}\right)\left(y_{d}-y_{e i}\right)\right] ; \\
& V_{2}=2\left[\left(x_{c 1}-x_{d}\right)\left(x_{d}-x_{e i}\right)-\left(y_{e 1}-y_{d}\right)\left(y_{d}-y_{e i}\right)\right] ; \\
& V_{3}=\left(x_{d}-x_{e 1}\right)\left(2 x_{c 1}-x_{d}-x_{e 1}\right)+\left(y_{d}-y_{e 1}\right)\left(2 y_{c 1}-y_{d}-y_{e 1}\right)-\left(x_{d}-x_{e i}\right)^{2}-\left(y_{d}-y_{e i}\right)^{2} .
\end{aligned}
$$

\section{Improved particle swarm optimization}

In view of the shortcomings of the basic particle swarm optimization algorithm, scholars at home and abroad have made some improvements on the velocity and position updating formula, particle randomness and population network structure, the improved results of velocity update formula is the most abundant. The velocity update formula is composed of original velocity, cognitive part and social part, of which the original speed is used to balance the global and local search ability, the cognitive part is used to make the particles have enough global searching ability, the main function of them is to avoid the particles fall into local minimum value [3]. In the basic particle swarm optimization algorithm, each iteration of particle is compared with the optimal value of the individual. This paper presents a global variation updating formula with step decreasing instead of chaotic mutation and the original cognitive speed. It is more emphasis on social part of the particle, and it greatly reduces the calculation complexity and improves the efficiency of the algorithm. Chaos is defined as a random access channel, which has the characteristics of ergodicity and randomness. The Logistic chaotic system is chosen as the variation of the chaoticvariation: 


$$
\left\{\begin{array}{c}
r(1) \in[0,1] \\
r(n+1)=\mu r(n)(1-x(n))
\end{array}\right.
$$

Among them, $r(n)$ is the $\mathrm{N}$ iteration value of the variable $\mathrm{R}, \mu$ is a constant in the interval $[1,4]$. In the early iterations, the algorithm is expected to have a larger mutation step, the diversity of particles is larger, the global search ability. While in later iterations, the smaller step size ensures the accuracy and efficiency of local search, therefore, in this paper, we add an attenuation factor which decreases with the number of iterations before the chaotic variation. The global mutation update formula is:

$$
x_{i j}(t+1)=x_{i j}(t)+c\left[g_{j}(t)-x_{i j}(t)\right]+a(t) r(t)
$$

In formula, $\quad x_{i j}(t) \quad$-- The $t$ iteration of the first $i$ particles in the position of the $j$ dimension

$c \quad$-- Learning factor

$g_{j}(t) \quad--T h e j$ value of the global optimal position

$a(t) \quad$--Attenuation factor

\section{Verification and analysis of optimization results for pulse generator}

The motion analysis of the original Zero-Max type continuously variable transmission is carried out, and the relationship between the input and output angular functions is obtained by using the Chebyshev interval method.Set up the improved particle swarm algorithm population size $\mathrm{N}=50$, iteration number $\mathrm{T}=80, c=1.494, \lambda(t)=1.2 * 10^{t}, a(t)=0.2 * 0.95^{t}$. In order to test the performance of the improved particle swarm algorithm, the basic particle swarm optimization algorithm is compared with the penalty value. They are independently run 30 times, the performance of statistical results are shown in Table 1, fitness, diversity and iterative relationship shown in Figure 2, before and after the optimization of the connecting rod size is shown in Table 2, before and after the optimization of the performance comparison is shown in Figure 3. Among them, the diversity is the evaluation index of the degree of particle dispersion, and the larger diversity means exploring larger space. In this paper, the formula is calculated according to the literature.

Table 1 statistical results

\begin{tabular}{cccccc}
\hline Algorithm & $\begin{array}{c}\text { Best } \\
\text { Value }\end{array}$ & $\begin{array}{c}\text { Average } \\
\text { Value }\end{array}$ & $\begin{array}{c}\text { Standard } \\
\text { Deviation }\end{array}$ & $\begin{array}{c}\text { TIME } \\
/(\mathrm{s})\end{array}$ & $\begin{array}{c}\text { Success } \\
\text { Rate }\end{array}$ \\
\hline $\begin{array}{c}\text { Improved } \\
\text { PSO }\end{array}$ & 0.0539 & 0.0678 & 0.0188 & 4.13 & $30 / 30$ \\
Basic PSO & 0.2898 & 0.6624 & 0.2481 & 17.52 & $28 / 30$ \\
\hline
\end{tabular}
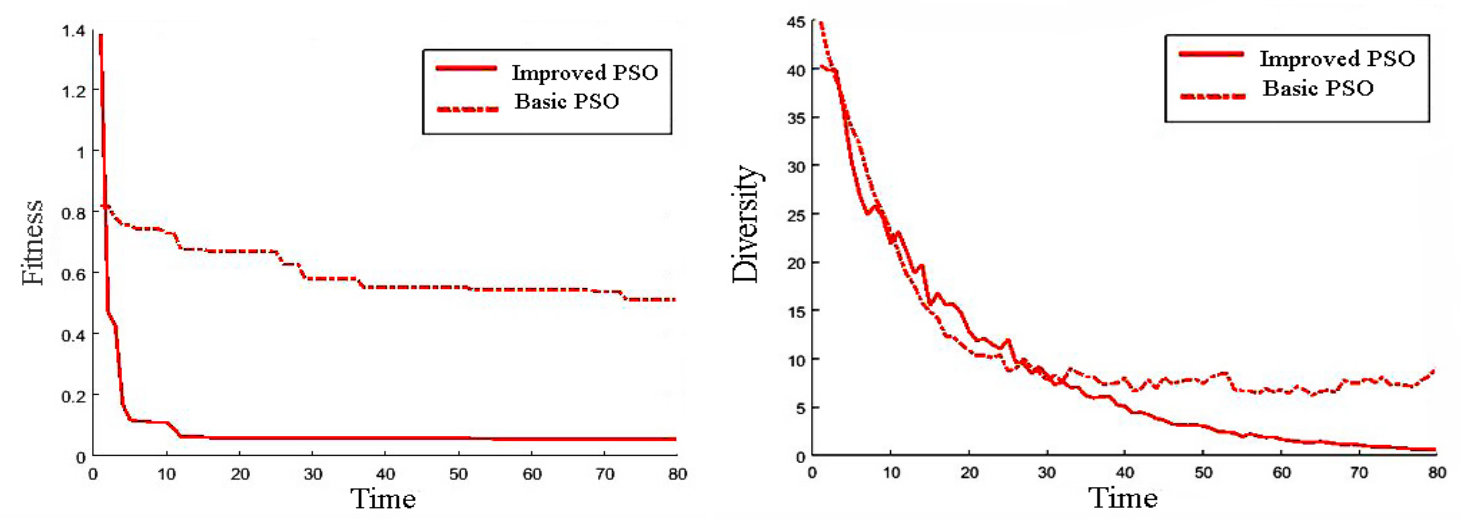

Fig. 2 the Schematic Diagram of Zero-Max CVT

As shown in Table 1, compared with the basic particle swarm optimization algorithm, the improved particle swarm optimization improves the accuracy and robustness by an order of magnitude, while the efficiency is improved by $70 \%$. According to figure 2 it shows that the improved particle swarm 
algorithm in the early iteration, diversity and diversity of basic particle swarm algorithm are similar, the size of space exploration is basically the same, but in later iterations, the diversity of improved particle swarm algorithm is convergent, the local search ability is stronger, and the search precision is improved.

Table 2 Rod Size

\begin{tabular}{ccccccc}
\hline Algorithm & $l_{1} /(\mathrm{mm})$ & $l_{2} /(\mathrm{mm})$ & $l_{3} /(\mathrm{mm})$ & $l_{4} /(\mathrm{mm})$ & $l_{5} /(\mathrm{mm})$ & $l_{6} /(\mathrm{mm})$ \\
\hline Before Optimization & 25 & 105 & 130 & 205 & 75 & 145 \\
After Optimization & 21.2 & 138.6 & 189.0 & 153.7 & 74.7 & 188.5 \\
\hline
\end{tabular}

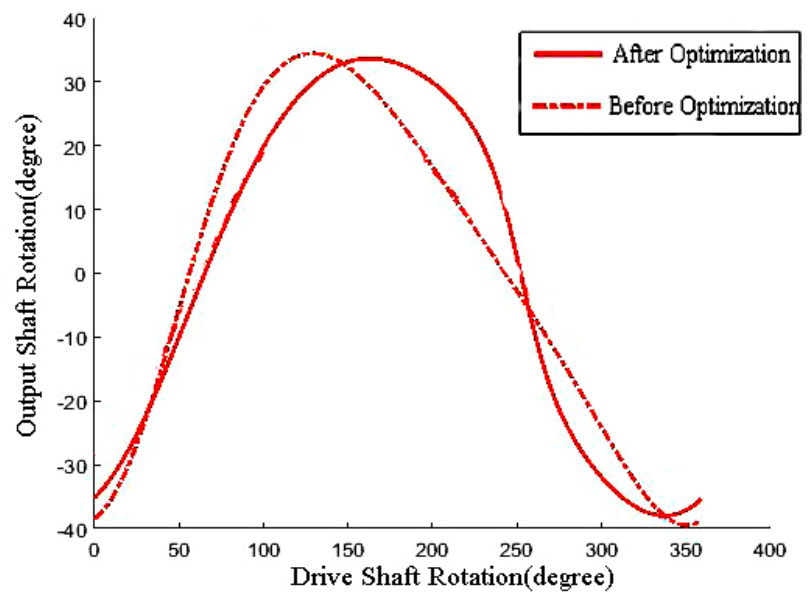

Fig. 3 the Motion Performance of the CVT

In addition, the motion performance of the mechanism is analyzed. As can be seen from Table 2, the change of the size of the overrunning clutch is also within the allowable value, which shows the validity of the penalty function method. The curve of Figure 3 is superposed by the phase difference of 90 degrees, and it is known that the pulsation of the original mechanism is about 0.32 , and the degree of fluctuation is reduced by a factor of 0.188 , which is reduced by about $41 \%$. After optimization, the mechanism has a more rapid response in the non-working area, which increases the time of the working area and the curve of the maximum value is smoother.

\section{Summary}

(1) The global mutation update formula and penalty function can effectively improve the accuracy and efficiency of PSO algorithm, which provides a new idea for the improvement of particle swarm optimization algorithm. The effectiveness of the improved particle swarm optimization algorithm is proved by the example of engineering optimization.

(2) The evaluation method of the single phase pulsation of the pulse CVT is proposed. The hinge point coordinate is taken as the decision variable, and the fluctuation is the evaluation index. The six - bar mechanism function is considered as the objective function of the algorithm, and the mathematical model is established by combining the length constraint and the transmission angle constraint.

\section{References}

[1] Dong Song,Songhui Nie. Kinematics simulation of Zero-Max type CVT based on SIMULINK. Machinery Design \& Manufacture, 2007(4):61-63.

[2] Songhui Nie. Principle and Characteristics for Original Non-Frictional Mechanical Continuously Variable Transmission. Chinese Journal of Mechanical Engineering , 2007, 43(9):190-194. 
[3] Jiandong, Wenyu, Hong, et al. Rotational Swashplate Pulse Continuously Variable Transmission Based on Helical Gear Axial Meshing Transmission. Chinese Journal of Mechanical Engineering, 2012, 25(6):1138-1143. 